\title{
Liquid layer flow over convex corners in the presence of an electric field
}

DOI:

$10.1063 / 1.4825475$

Link to publication record in Manchester Research Explorer

\section{Citation for published version (APA):}

Farid, S., \& Gajjar, J. S. B. (2013). Liquid layer flow over convex corners in the presence of an electric field. In AIP Conference Proceedings/AIP Conf. Proc. (Vol. 1558, pp. 277-280). American Institute of Physics.

https://doi.org/10.1063/1.4825475

\section{Published in:}

AIP Conference Proceedings|AIP Conf. Proc.

\section{Citing this paper}

Please note that where the full-text provided on Manchester Research Explorer is the Author Accepted Manuscript or Proof version this may differ from the final Published version. If citing, it is advised that you check and use the publisher's definitive version.

\section{General rights}

Copyright and moral rights for the publications made accessible in the Research Explorer are retained by the authors and/or other copyright owners and it is a condition of accessing publications that users recognise and abide by the legal requirements associated with these rights.

\section{Takedown policy}

If you believe that this document breaches copyright please refer to the University of Manchester's Takedown Procedures [http://man.ac.uk/04Y6Bo] or contact uml.scholarlycommunications@manchester.ac.uk providing relevant details, so we can investigate your claim.

\section{OPEN ACCESS}




\title{
Liquid Layer Flow over Convex Corners in the Presence of an Electric Field
}

\author{
Saadia Farid and Jitesh S.B. Gajjar \\ School of Mathematics, University of Manchester, Manchester M13 9PL, UK
}

\begin{abstract}
The behaviour of a thin layer of liquid when it flows over a convex corner and in the presence of an electric field, is studied in the context of triple-deck theory. It is assumed that the Reynolds number is large. The same problem without the presence of the electric field and surface tension effects was studied by [1]. The governing equations lead to a novel tripledeck problem and linear and nonlinear numerical solutions are obtained for various limiting cases of the Weber number and capillary number.
\end{abstract}

Keywords: Boundary layer, high Reynolds number, triple-deck, liquid layer, electric field

PACS: $47.15,68.15,73.61$

\section{INTRODUCTION}

Thin film flows play a significant role in several areas of engineering, geophysics and biophysics and have important application in for example, nanofluidics and microfluidics, coating flows. These flows have been the subject of much attention recently in the literature, and the theory and many applications are discussed extensively in [2]. In recent work the behaviour of liquid layer flows under the influence of an electric field has also been studied, see for example Tseluiko \& Papageorgiou [3]. But in many of these papers it is assumed that the Reynolds number is small. The aim of current work is to study the behaviour of a liquid layer in the presence of a uniform electric field, but taking the Reynolds number to be large. Two other dimensionless parameters, the electric Weber number (We) and the capillary number $(\mathrm{Ca})$ also arise in this study. The electric Weber number occurs due to the contribution of the electric field and will be taken to be large also, and the capillary number $\mathrm{Ca}$ is assumed to be small. The precise scaling for the different parameters is discussed later. Liquid layer flow over a convex corner in absence of an electric field has already been discussed in [1] where surface tension effects were also ignored. The present problem is a modification of that of [1] by introducing an electric field and keeping surface tension effects.

\section{DESCRIPTION OF THE PROBLEM AND ANALYSIS}

We consider the steady, laminar, two-dimensional flow of a liquid layer of constant density $\rho$ and viscosity $\mu$. The liquid flows under gravity along an infinitely long plate which is inclined at angles $\beta^{*}$ and $\beta^{*}+\alpha^{*}$ to the horizontal upstream and downstream respectively (as shown in Fig. 1). The oncoming profile is fully developed and the Reynolds number $(R)$ is assumed to be large throughout. The surface tension coefficient between the liquid and surrounding medium is $\sigma$ and $g$ denotes the acceleration due to gravity. The plate is taken to be an infinite electrode which, without loss of generality, is held at zero voltage potential. Far from the plate, there is a uniform electric field $E_{0}$ which is normal to the plate. The surrounding medium (Region II) is assumed to be a perfect dielectric with permittivity $\varepsilon_{a}$. In this problem the liquid is assumed to be a perfect conductor which implies that the potential on the deformed liquid free surface is zero (the electric field in the liquid is zero).

The governing equations for this flow are the Navier-Stokes equations for the liquid layer supplemented by Laplace's equation for the electric potential, see [4]. The boundary conditions are of no slip at the plate and continuity of normal and tangential stresses at the free surface together with the kinematic condition at the free surface. The key contributions due to capillary effects and Maxwellian stresses produced by the presence of the electric field arise in the boundary conditions.

We take a set of Cartesian axes $O x^{*}, z^{*}$, see Fig. 1. We will denote the liquid layer by Region I and the surrounding medium by Region II. The basic flow far upstream is given by velocity $\left(U_{B}^{*}, W_{B}^{*}\right)$, pressure $P_{B}^{*}$ and electric potential $V_{B}^{*}$.

11th International Conference of Numerical Analysis and Applied Mathematics 2013

AIP Conf. Proc. 1558, 277-280 (2013); doi: 10.1063/1.4825475

(C) 2013 AIP Publishing LLC 978-0-7354-1184-5/\$30.00 


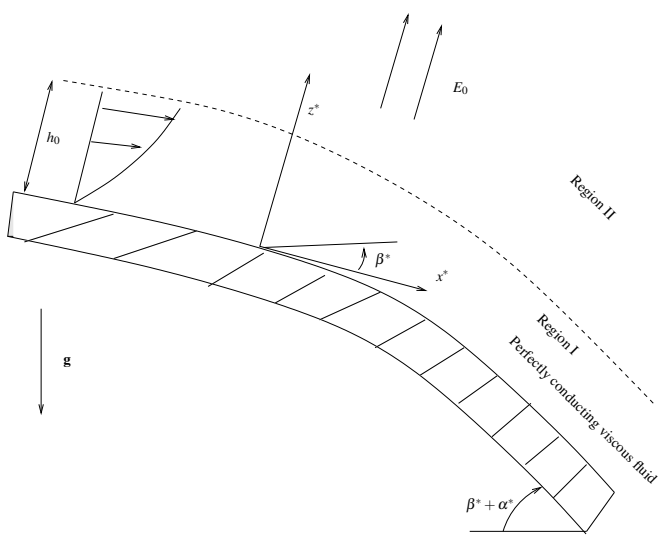

FIGURE 1. Liquid layer near a convex corner in presence of normal electric field. The dashed line denote the free surface.

There is an exact solution far upstream which is analogous to the Nusselt solution for the non-electric case [5], and has $z^{*}=h_{0}$ with $h_{0}$ being the undisturbed depth of the liquid layer far upstream. The exact solution is given by

$$
\begin{gathered}
U_{B}^{*}=\frac{g \sin \beta^{*}}{2 v}\left(h_{0}^{2}-\left(z^{*}-h_{0}\right)^{2}\right), \quad W_{B}^{*}=0, \\
P_{B}^{*}=p_{o}^{*}-\rho g\left(z^{*}-h_{0}\right) \cos \beta^{*}-\frac{1}{2} \varepsilon_{a} E_{0}^{2}, \quad V_{B}^{*}=-E_{0}\left(z^{*}-h_{0}\right) .
\end{gathered}
$$

This gives an electric field $\mathbf{E}_{B}^{*}=-\nabla V_{B}^{*}=\left(0, E_{0}\right)$.

The solution upstream is modified due to the presence of the corner. Taking the Reynolds number to be large, one can perform an asymptotic analysis perturbing about the exact solution far upstream. Following [6], [1] even very small angles induce significant transverse pressure gradients acting across the whole layer and over long length scales. As our starting point we take the same scalings as in [1] and ask what are the crucial scalings for the electric field and surface tension which modify this problem. We define the Reynolds number $R$, the Capillary number $C a$, and the Weber number $W e$ by

$$
R=\frac{U_{c} h_{0}}{v}=\frac{g h_{0}^{3} \sin \beta^{*}}{v^{2}}, \quad C a=\frac{U_{c} \mu}{\sigma}=\frac{\rho g h_{0}^{2} \sin \beta^{*}}{\sigma}, \quad W e=\frac{\varepsilon_{a} E_{0}^{2}}{\rho g h_{0} \sin \beta^{*}},
$$

where $v=\frac{\mu}{\rho}$ is kinematic viscosity. Introducing a small parameter $\varepsilon=R^{-\frac{1}{7}}$ significant changes to the non-electric case discussed in [1] arise when the Weber number is large and the Capillary number small. Specifically we take

$$
W e=\varepsilon^{-6} W_{e}, \quad \text { and } \quad C a=\varepsilon^{7} C,
$$

with $W_{e}$, and $C$ to be $O(1)$.

Details of the analysis are omitted here but can be found in [1], [7] and this shows that the perturbed flow in the liquid layer is governed by the following interactive boundary layer equations written in non-dimensional, scaled, form as:

$$
\begin{gathered}
U_{X}+V_{Y}=0, \\
U U_{X}+V U_{Y}=-P_{X}+U_{Y Y}, \\
0=P_{Y},
\end{gathered}
$$

with boundary conditions

$$
\begin{gathered}
U=V=0 \quad \text { on } \quad Y=0, \\
U \rightarrow Y+A-\alpha F(X) \quad \text { as } \quad Y \rightarrow \infty,
\end{gathered}
$$


a)

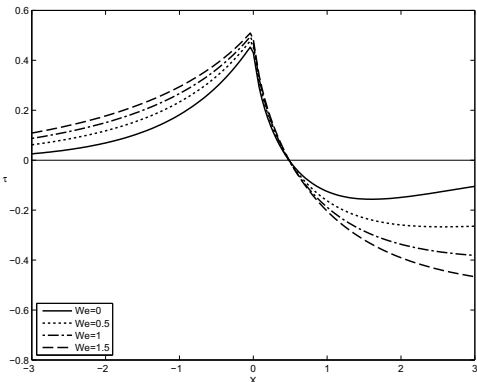

(b)

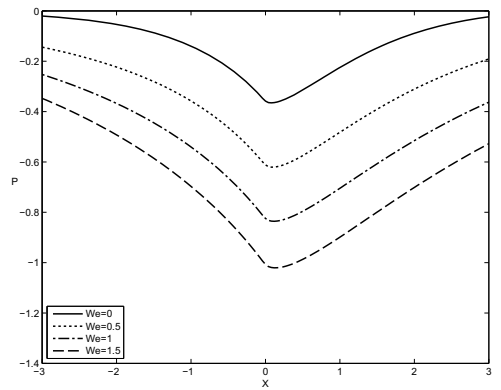

FIGURE 2. Plots of the perturbation (a) skin friction $\tau$ and (b) the pressure, for the case $s=0, C=10$ and varying $W_{e}$

$$
U \rightarrow Y \quad \text { as } \quad X \rightarrow-\infty
$$

and interaction law,

$$
P=-s A-A^{\prime \prime}+\frac{W_{e}}{\pi} f_{-\infty}^{\infty} \frac{A^{\prime}(\xi)}{X-\xi} d \xi+\frac{1}{C} A^{\prime \prime} \quad, \quad \eta=-A .
$$

Here $(U(X, Y), V(X, Y))$ are the velocity components in terms of scaled $(X, Y)$ coordinates with $Y$ reflecting the effect of a Prandtl transformation. Also $P(X)$ is the induced pressure, $\eta(X)=-A(X)$ is the scaled perturbed free-surface, and $F(X)$ represents the scaled wall shape function. For a sharp corner we take $F(X)=X H(X)$ with $H(X)$ being the Heaviside unit step function. Instead of a corner the analysis can also cover shapes such as localised humps by taking a suitable form for $F(X)$. The parameter $\alpha$ is a scaled parameter representing the amount of deviation from a straight wall, and the parameter $s$ is as in [1], and gives the relative importance of the induced hydrostatic pressure gradient. If $W_{e}=0$, and $C \gg 1$ the above reduces to the problem discussed by [1].

\section{RESULTS AND DISCUSSION}

The novel interaction law 7 is the key result stemming from this analysis and show that the influence of the electric field is non-local. The interaction law is a combination of that occurring in hypersonic and jet flows as well as in subsonic flow. For large $W_{e}$ the problem is the same as that for incompressible, or subsonic, triple-deck flow over a convex corner as studied by [8]. This suggests that for increasing $\alpha$ the presence of the electric field will lead to flow separation in the vicinity of the corner as with the subsonic triple-deck flow over corners.

Numerical solutions of the above triple-deck problem are currently in progress. For small $\alpha$ a linearised analysis can be conducted. In Fig. 2 - Fig. 4 we have shown sample results for three cases which may be compared with the results for the non-electric case given in [1] for a sharp corner. In Fig. 2 results are displayed for the perturbation skin friction $\tau(X)$ and perturbation pressure $P(X)$ with $s=0, C=10$ with $W e$ varying. The presence of the electric field causes the flow to accelerate ahead of the corner faster than for the non-electric case and the skin friction falls much more rapidly downstream of the corner. In Fig. 2 the pressure reduces much more ahead of the corner before recovering downstream of the corner, as compared to the non-electric case. Results for $s=0.2$ in Fig. 3 and $s=1$ in Fig. 4 keeping the other parameters the same, also show similar trends. This would tend to suggest that the presence of a strong electric field is much more likely to promote separation in the vicinity of the corner in comparison to the non-electric case. The presence of localised regions of flow separation does imply significant differences in the flow properties as compared to studies at low Reynolds numbers where flow separation is ignored.

\section{ACKNOWLEDGMENTS}

Farid gratefully acknowledges the financial support of the University of Engineering and Technology, Lahore, for her studies at the University of Manchester. 
a)

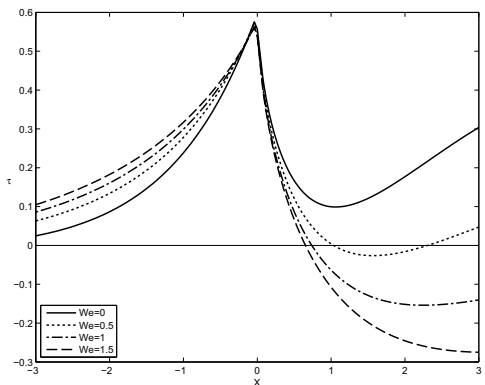

(b)

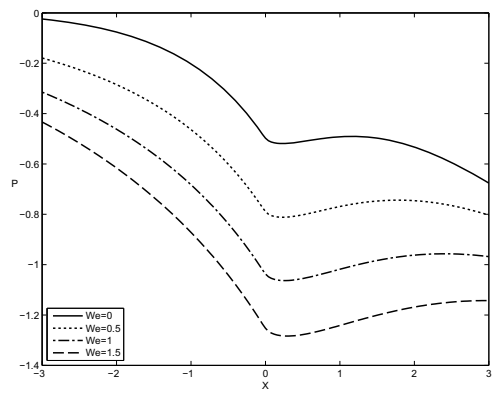

FIGURE 3. Plots of the perturbation (a) skin friction $\tau$ and (b) the pressure, for the case $s=0.2, C=10$ and varying $W_{e}$

a)

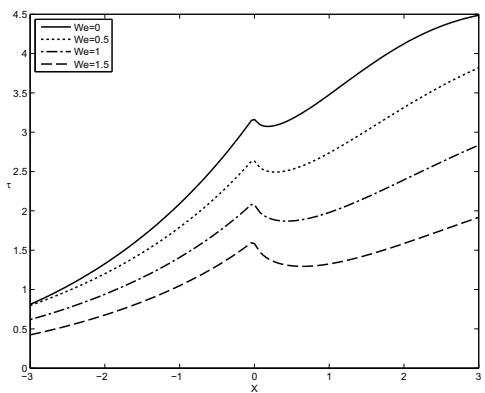

(b)

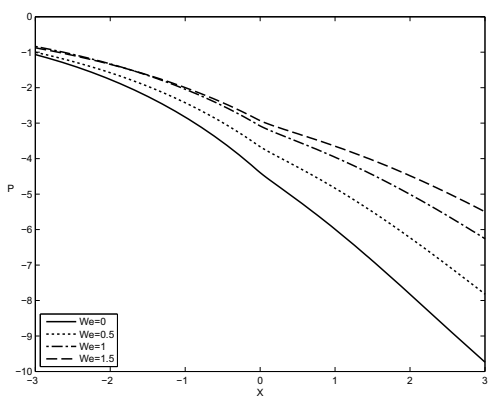

FIGURE 4. Plots of the perturbation (a) skin friction $\tau$ and (b) the pressure, for the case $s=1.0, C=10$ and varying $W_{e}$

\section{REFERENCES}

1. Gajjar, J.S.B. (1987), Fully developed free surface flows - Liquid layer flow over a convex corner. Computers \& Fluids 15 , 337-360.

2. Craster, R.V. \& Matar, O.K. (2009), Dynamics and stability of thin liquid films. Reviews of Modern Physics 81, 1131-1198.

3. Tseluiko, D. \& Papageorgiou, D.T. (2006), Wave evolution on electrified falling films. J. Fluid Mech. 556, 361-386.

4. Tseluiko, D., Blyth, M.G., Papageorgiou, D.T., and Vanden-Broeck J.-M (2009) Viscous electrified film flow over step topography. SIAM J. Appl. Math., 3, 845-865.

5. Benjamin, T. B. (1957), Wave formation in laminar flow down an inclined plane.J. Fluid Mech. 2, 554, $226-574$.

6. Smith F.T. (1977), Upstream interactions in channel flows. J. Fluid. Mech. 79, 631-655

7. Farid, S. (2013), Ph.D. Thesis, University of Manchester (in preparation).

8. Stewartson, S. (1970), On laminar boundary layers near corners. Q.J. Mech. Appl.Math., 14, 145-239. 